\title{
Assessing emergence from a Prolonged Disorder of Consciousness: current opinion and practice in the UK
}

\author{
Amy Pundole \\ Division of Psychology and Language Sciences, University College London, UK. \\ Clinical Lead Speech and Language Therapist, Royal Hospital for Neurodisability, London, UK \\ Professor Rosemary Varley \\ Division of Psychology and Language Sciences, University College London, UK. \\ Dr Suzanne Beeke \\ Division of Psychology and Language Sciences, University College London, UK.
}

\begin{abstract}
Background: A patient in a prolonged disorder of consciousness (PDOC) is currently required to demonstrate functional object use or functional communication to be considered to have emerged from this state. A range of tasks and stimuli are used in assessment and patients are expected to achieve $100 \%$ accuracy. As consciousness is considered to occur along a continuum, determining emergence is not straightforward.
\end{abstract}

Objective: To establish the opinions of expert clinicians on the criteria and tasks recommended for determining emergence from PDOC, and how emergence is determined in practice. This will identify current issues and define objectives for further research.

Methods: A 31 item pilot-tested survey was distributed electronically to multidisciplinary teams working in post-acute specialist rehabilitation settings across the UK. Survey items included questions on diagnosis and confidence, informal assessment, formal assessment, and family involvement. Descriptive statistics were used to analyse responses to closed questions. Participant responses to open questions were analysed using thematic analysis (Braun \& Clarke, 2006).

Results: One hundred clinicians from a range of professions completed the survey. Approximately a third of respondents (30.4\%) used tasks other than those recommended by Giacino et al. (2002) and the Royal College of Physicians Guidelines (RCP, 2013) to determine emergence. A lack of confidence in the current tasks used to specifically detect the return of functional communication was reported by $46.4 \%$ of respondents. The majority of respondents $(78.6 \%)$ reported that they worked with patients who they considered to have emerged, but who were unable to demonstrate emergence based on the current criteria. There was differing frequency in use of recommended tasks to identify functional communication: $100 \%$ used yes/no tasks, $88.9 \%$ used discrimination tasks, and $55.6 \%$ used matching tasks. A range of different stimuli were employed, but $30.6 \%$ of respondents were not confident that they were able to choose stimuli appropriately. Respondents reported a 
range of benefits and challenges when involving family and friends in the assessment of emergence.

Conclusion: This study highlights the difficulties encountered by experienced clinicians in the UK when using the current recommended tasks and criteria to determine when a patient has emerged from PDOC. Accurate assessment and diagnosis are vital as they provide information about prognosis and influence decisions about ongoing care and rehabilitation. It would be beneficial to review the emergence criteria and determine which tasks and stimuli are most appropriate for assessment.

Keywords: Prolonged Disorder of Consciousness (PDOC), emergence, severe cognitive impairment, assessment, family.

\section{Introduction}

A disorder of consciousness can occur following severe brain injury and is considered prolonged if persisting for longer than four weeks (PDOC). Patients may progress along the continuum of consciousness, through the stages of Coma, Vegetative State (VS) and Minimally Conscious State (MCS) before emerging (The Royal College of Physicians (RCP) National Clinical Guidelines on Prolonged Disorders of Consciousness, 2013). Patients in VS have a sleep-wake cycle and display a range of reflexive and spontaneous behaviours, but do not demonstrate any evidence of awareness of themselves or the environment (RCP guidelines, 2013, Multi Society Task Force on Persistent Vegetative State 1994). MCS is characterised by inconsistent, but reproducible responses above the level of spontaneous or reflexive behaviour, which indicate some awareness of the environment (Giacino et al., 2002).

Giacino et al. (2002) defined emergence from MCS and produced guidance as to how it should be assessed. Emergence was defined as the demonstration of reliable functional communication or the functional use of two different objects. However, determining the boundary between MCS and emergence is challenging as the current criteria focus on a narrow range of cognitive abilities that require $100 \%$ performance accuracy (Pundole \& Crawford, 2017). To facilitate consistent practice in the assessment of emergence, there is specification of tasks and scoring criteria. Giacino et al. (2002) define functional object use as appropriate use of two objects on two consecutive evaluations, e.g., bringing a comb to the head, Functional communication is recognised when a person answers $6 / 6$ situational yes/no orientation questions accurately on two consecutive occasions, e.g., "Am I pointing to the ceiling?" Responses can be verbal, written, gestured or provided using a communication device. Reliability and accuracy of response were highlighted as the defining features of emergence. Responses that remain unreliable are considered consistent with MCS. However, given that this patient group have suffered significant brain injury, even a patient who has emerged from a disorder of consciousness will have ongoing language and/or non-language cognitive impairments that may prevent them achieving $100 \%$ accuracy in these tasks 
(Dymowski et al., 2015; Kohl et al. 2009; LeBlanc et al., 2006; McDonald et al., 2014; Russell et al., 2012; Schnakers et al., 2015; Slovarp et al., 2012).

If a patient emerging from PDOC is unable to demonstrate functional communication or functional object use to the required performance levels, they may be categorised as MCS even if they demonstrate evidence of consistent awareness of self and environment via a range of behaviours. The RCP guidelines (2013) explicitly acknowledge that misdiagnosis of emergence is possible, but state that if there is any uncertainty then the individual should be categorised as remaining in a MCS, as patients in PDOC in England should receive non-means tested NHS continuing health care $(\mathrm{CHC})$ funding as a priority. However, if a patient is misdiagnosed as MCS, they may be less likely to receive ongoing rehabilitation, as resources are targeted where most change is expected (Samanta et al. 2016). Furthermore, Clinicians and families need to consider level of functioning, prognosis and what quality of life would be acceptable to the patient when making decisions in an individual's best interests, in accordance with the Mental Capacity Act (2005), in the absence of a Lasting Power of Attorney for Health, or Advanced Decision to Refuse Treatment

Families often report behaviours that are not apparent to clinicians, and while they can sometimes misinterpret how meaningful these are, there is evidence that patients may respond differently to familiar people (Barreca et al., 2003; Sattin et al., 2014). If the views of family differ from those of clinicians, there is potential for tension in the relationship between the family and team, which can impact on the process of collaborative decision making about future care.

This focus on accurate yes/no responses or object use to indicate emergence disadvantages patients in several ways. First, patients with a severe motor deficit are only be able to indicate consciousness by accurately responding to yes/no questions, since object manipulation is impaired. Second, patients with severe language impairments will not be able to achieve the accuracy of response required for yes/no tasks. Given the profound nature of their impairments, patients may need to learn an alternative yes/no response and recall how to use it (e.g., eyes up for yes, versus down for no, or eye pointing to written yes/no cards). This further complicates the task since, in addition to understanding the question, the response strategy must be recalled and implemented while maintain the question and response in working memory. Furthermore, patients who have emerged from a disorder of consciousness have been found to have specific difficulty with yes/no responses (Nakase-Richardson et al., 2008). Some patients with severe receptive and expressive language impairment (global aphasia) have been unable to demonstrate functional communication, so fail to meet the criteria for emergence despite not being in PDOC (Schnakers et al., 2015). As patients emerging from PDOC are likely to have a severe language and/or non-language cognitive impairment, they risk being misdiagnosed as MCS.

Despite Giacino et al.'s (2002) attempts to specify stimuli, a range of yes/no questions have been observed in practice and reported in the research literature. Question types include those that are autobiographical (Is your name John?), situational (Am I clapping right now?), and those that involve a perceptual judgement (Is this a cup?). Nakase-Richardson et al., (2009) demonstrated that following a traumatic brain injury, even patients, who have clearly 
regained consciousness found situational and judgement questions difficult to answer. Some questions that are related to the self and to familiar stimuli appear to facilitate a response (Abbate, et al., 2014; Sun et al., 2018). Furthermore, although family contribution to the assessment of emergence is not well defined, it can have a positive impact on outcome, improving diagnosis from MCS to emerged in some cases (Sattin et al., 2014).

To address some of the concerns about yes/no questions, the RCP guidelines (2013) included additional tasks to assess for emergence: discrimination between two objects or pictures, and matching pairs of objects. Discrimination refers to spoken word-to-picture/object matching in the presence of a distractor, while object matching involves three items being placed where the patient can see them, attend to each and then indicate their response. It may therefore be more challenging to present than yes/no questions or discrimination of two items. As many as $65 \%$ of patients in PDOC have visual problems, either pre-existing or associated with their brain injury (Andrews, Murphy, Munday, \& Littlewood, 1996), which could impact on their ability to attend to, scan and process the visual information required for this task. Patients are again required to achieve $100 \%$ accuracy on trials. For the discrimination task, the nature of the distractor is only loosely specified. 'Cat versus another animal' is suggested, but such items are semantically similar and could be visually similar, e.g. cat versus dog, which could be more difficult for cognitively-impaired patients to discriminate. Extending the tasks used to assess for emergence is useful, but it would be beneficial to closely specify target and distractor items in order that assessment of emergence is not confounded by impairments of linguistic or visual processing. This could include ensuring target items are of high lexical frequency, and are visually, phonologically and semantically distinct from their distractor.

In addition to the specific tasks linked to emergence criteria, three formal assessments are recommended by the RCP (2013) for the assessment of patients in PDOC. These are the Sensory Modality Assessment and Rehabilitation Technique (SMART, Gill-Thwaites, 1997); the Coma Recovery Scale-Revised (CRS-R, Giacino, Kalmar, \& Whyte, 2004) and the Wessex Head Injury Matrix (WHIM, Shiel et al., 2000). The latest update of SMART (2019) includes optional emergence tasks that are explicitly based on the RCP guidelines (2013). They include functional object use, situational or biographical yes/no questions, matching pairs, and discrimination between two objects or pictures. Assessment of emergence by the CRS-R is specifically aligned to Giacino et al.'s (2002) criteria of functional object use and correct responses to situational yes/no questions. The WHIM was not designed as a PDOC assessment so does not explicitly assess emergence. However, a small number of test items are relevant, for example 'points with eyes between a choice of two pictures or real objects or 'yes/no' cards correctly on 9/10 trials' (item 47).

In view of challenges in assessing emergence, the variety of tasks employed, and the lack of closely controlled stimuli, this study surveyed specialist clinicians across the UK who are experienced in assessing emergence from PDOC. The aim was to ascertain their opinions and practice when assessing for emergence. No similar surveys examining this area of clinical assessment have been published.

\section{Method}


The reporting of this survey conforms to the Checklist for Reporting Results of Internet ESurveys (CHERRIES) (Eysenbach, 2004; Turk et al., 2018). Questions were devised by the authors based on the background literature, existing guidelines and clinical experience. Participants were asked a range of questions about their current practice and their opinions on the recommended criteria, tasks and assessments. This included questions on what stimuli they use for different tasks, how they include family in assessment and how they make decisions about diagnosis if they believe a patient is no longer in a disorder of consciousness but does not meet the current criteria for emergence. The survey was categorised as service evaluation according to NHS Health Research Authority guidance and institutional ethical approval was not required.

Five clinicians drawn from a range of professions (occupational therapy (OT) physiotherapy (PT), neuropsychology (Psych), and speech and language therapy (SLT)) piloted the initial 28 item survey to ensure questions and format were appropriate and unambiguous. These clinicians were experienced in assessing PDOC patients, but worked in a nursing home setting so were not eligible to complete the final survey. Pilot respondents recorded the time taken to complete the survey and provided written feedback on the format, category fields and wording of questions. Modification in response to feedback included rewording some of the questions and adding three questions to specifically assess confidence in administering recommended tasks.

The final survey included 31 questions divided into five sections: (1) background information; (2) confidence in diagnosis of emergence; (3) assessment of functional communication to determine emergence; (4) formal assessment of emergence; (5) family involvement in assessing functional communication to determine emergence. A combination of closed questions (for speed of completion) and open questions (to elicit additional information), were included (Schaeffer \& Dykema, 2011). Questions are provided in appendix I.

Questions were presented in fixed order and a response had to be entered before the next question was shown. A back button allowed responses to be reviewed and a progress bar was provided. Adaptive questioning was employed so certain items were only displayed dependent on previous response. This reduced the number of questions for some respondents. The initial page of the survey included a statement making it explicit that by continuing respondents were giving consent to participate in the survey and that their responses would be stored anonymously and securely. No personal data were collected. The survey was designed and implemented with Opinio 7.10 software.

The survey was targeted at experienced teams, who regularly admit medically stable patients in PDOC for assessment, diagnosis and management in a post-acute setting. Clinicians also assess emergence in PDOC patients in other settings (e.g., acute and continuing care). However, the RCP Guidelines (2013) suggest that acute teams may not always have specialist knowledge and experience of working with PDOC as they have different priorities, such as medical stabilisation, airway management and establishing adequate nutrition. For these reasons, the survey was only targeted at teams in post-acute settings.

A list of 24 Level 1 and Level 2 centres in England was obtained from the UK Rehabilitation Outcomes Collaborative (UKROC). There is no record of centres in Wales, Scotland or 
Northern Ireland, so a Freedom of Information request was submitted to 26 Health Boards (Wales=7, Scotland=14, Northern Ireland=5) which identified a further nine possible centres. The first author contacted each of the 33 potential sites by telephone to determine if PDOC patients were regularly admitted, defined as at least three patients a year over the last 3 years. Fifteen centres across the UK were identified that met these criteria and were included in the survey. Information about geographical location was not collected in the final survey, as due to single centres serving some areas, this information might have identified participants.

A key contact at each centre was established by telephone and were primarily SLTs due to the focus on communication when determining emergence. Each contact was asked to disseminate an invitation email containing a link to the online survey to doctors and allied health professionals involved in assessment and final diagnosis decisions for PDOC patients. These clinicians were expected to be experienced in assessing emergence.

Contacts also reported the number of clinicians the invitation was sent to, so response rate could be determined. After the initial invitation, two reminders were sent to signal when the survey was active. The survey was open for 6 weeks during March and April 2019. No incentives were offered.

Data was downloaded to IBM's Statistical Package for Social Science Software version 25 (IBM Corp, 2017). Descriptive statistics were used to analyse responses to closed questions. Participant responses to open questions were analysed using thematic analysis to code responses and identify key themes (Braun \& Clarke, 2006). To ensure a rigorous approach, data were analysed using Braun and Clarke's (2006) analysis checklist, and the first and third authors met to discuss and finalise coding and themes.

\section{Results}

Fifteen services provided information as to the number of team members to whom the survey was sent. It was disseminated to 246 clinicians (mean 19 per setting). Two services did not provide this information. Filling the two missing data points using this mean, the total number of invitations sent was approximately 284 . One hundred respondents started the survey, giving a response rate of $35.2 \%$. The mean response rate where a survey is disseminated by key individuals within an organization, is $34 \%$ (Fulton, 2018). There were two questions in the survey designed to allow respondents to discontinue if they were not responsible for assessing emergence and more specifically functional communication. Thirty-nine respondents discontinued at these points. Twenty-five respondents completed some questions, but discontinued at random points. Thirty six completed the full set of questions. Data from respondents discontinuing randomly were not included for analysis. Therefore, data from 75 respondents were included in the final analysis.

\section{Characteristics of survey respondents}

A range of professionals responded, reflecting the interdisciplinary nature of working with patients emerging from PDOC. The largest group to respond were SLTs $(26.7 \%, n=20)$, as might be expected given the emphasis on communication behaviours both in the definition 
of emergence and the methods for assessing it. It is possible that identifying SLTs as centre contacts influenced respondent type, but a wide range of other professionals also submitted completed surveys. Table 1 displays the professional grouping of respondents whose data were analysed.

Most respondents had worked with PDOC patients for more than 2 years $(81.3 \% n=61)$ and almost a third had lengthy experience, having worked with PDOC patients for more than 10 years $(32.0 \%, \mathrm{n}=24)$. Most respondents worked in settings that admitted more than five PDOC patients a year $(82.7 \%, n=62)$, with $44.0 \%(n=33)$ working in highly specialist settings admitting more than 20 patients a year. Thus, the survey was completed by clinicians highly experienced in assessing patients emerging from PDOC.

Table 1: Characteristics of survey respondents $(n=75)$

\begin{tabular}{ll}
\hline Professional group & \\
Doctor (Dr) & $21.3 \%(16)$ \\
Nurse (RN) & $1.3 \%(1)$ \\
Occupational Therapist (OT) & $18.7 \%(14)$ \\
Physiotherapist (PT) & $17.3 \%(13)$ \\
Psychologist (Psych) & $14.7 \%(11)$ \\
Speech and Language Therapist (SLT) & $26.7 \%(20)$ \\
\hline Number of years working with PDOC patients & \\
$0-1$ & $18.7 \%(14)$ \\
$2-5$ & $30.7 \%(23)$ \\
$6-10$ & $18.7 \%(14)$ \\
$>10$ & $32.0 \%(24)$ \\
\hline Reported number of PDOC patients admitted to service per year & \\
$0-5$ & $17.3 \%(13)$ \\
$6-10$ & $13.3 \%(10)$ \\
$11-20$ & $25.3 \%(19)$ \\
$>20$ & $44.0 \%(33)$ \\
\hline
\end{tabular}

Nineteen of 75 (25.3\%) respondents exited the survey after the first four background questions, as they were not involved directly in assessing emergence. These were doctors $(47.4 \%, n=9)$ and nurses $(5.3 \%, n=1)$, who were primarily be involved in medical management and care, PTs $(36.8 \% \mathrm{n}=7)$ who were responsible for physical management, and psychologists $(10.5 \%, n=2)$. While psychologists often have a role in assessing awareness and cognition, some may assume a more consultative role or focus on supporting families.

A further 20 respondents (26.7\%) exited at question 14, as they were not specifically involved in assessing functional communication to determine emergence. The remaining 36 of 75 respondents completed the final 17 questions on assessing functional communication, and on family involvement (see Table 2). The greatest proportion of these were SLTs, $55.6 \%$ $(n=20)$, but other professionals were involved, most notably OTs, which may reflect their use of the SMART assessment, and an interdisciplinary approach to working with patients in PDOC. 
Table 2: Team members who are responsible for assessing emergence and functional communication

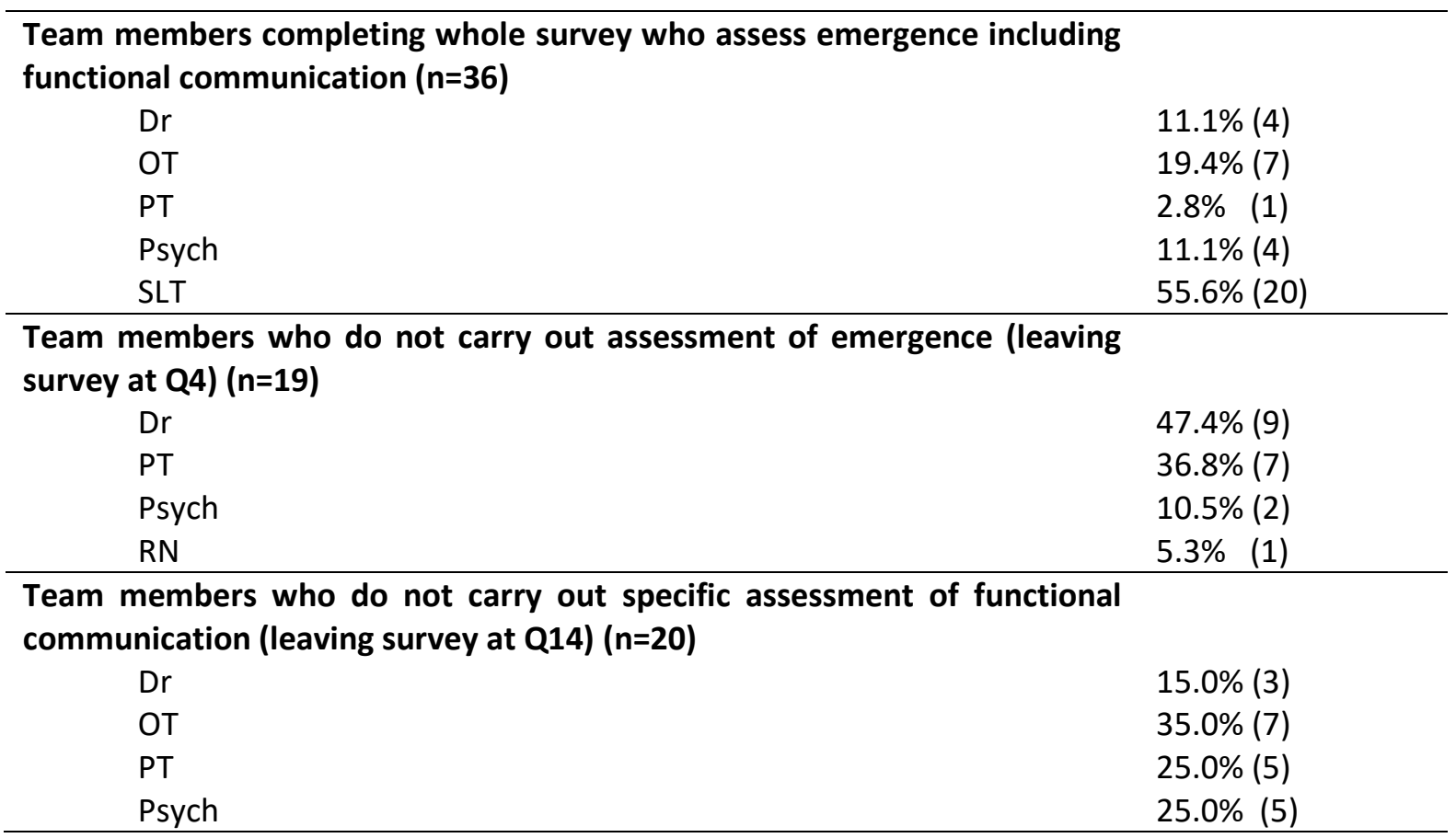

\section{Diagnosis and Confidence in current methods of assessment/criteria}

Fifty-six respondents were eligible to answer questions in this section. A diagnosis of emergence was a team decision according to $98.2 \%(n=55)$ of respondents. This reflects the RCP Guidelines (2013) which emphasise that diagnosis and management require a teambased approach.

While most respondents reported using the recommended tasks to determine if a patient had emerged from PDOC (69.6\%, $n=39)$, a third reported using others $(30.4 \%, n=17)$. This subgroup included a range of professionals ( $\operatorname{Dr} 17.6 \% n=3$, OT $23.5 \% n=4$, PT $11.8 \% n=2$, Psych $11.8 \% n=2)$, but the largest proportion were SLTs $(35.3 \%, n=6)$.

Clinicians who reported choosing other tasks had worked with PDOC patients for a range of years and reported their centre admitted a varying number of PDOC patients per year (Figures 1). Those with more than 10 years of experience of working with PDOC were most likely to use other tasks $(35.3 \%, n=6)$, although many less experienced clinicians also did this.

Figure 1: Number of years of experience of PDOC in respondents who reported using tasks other than those currently recommended to assess emergence. 


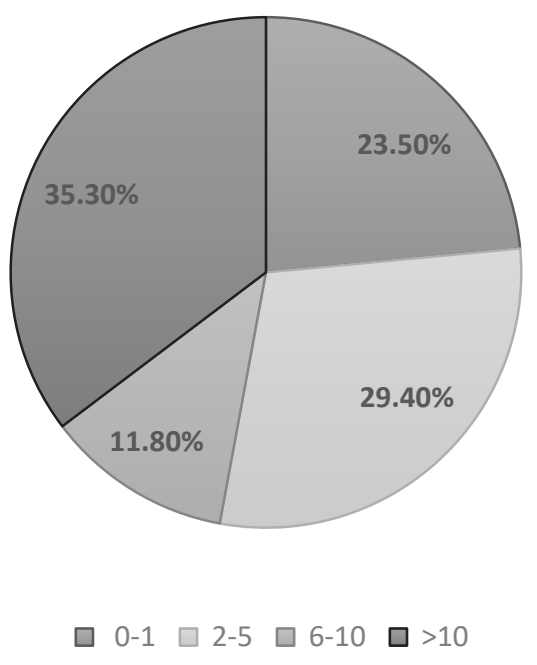

Respondents differed in degree of confidence in the recommended tasks used to determine emergence: $41.1 \%(n=23)$ reported confidence, while $42.9 \%(n=24)$ were not confident. However, only $21.4 \%(n=12)$ were confident that the recommended tasks specifically detect the return of functional communication, whereas $46.4 \%(n=26)$ did not have confidence in the tasks for this purpose.

The majority of respondents $(78.6 \%, \mathrm{n}=44)$ reported that they worked with patients who they thought had emerged but were unable to meet the current criteria, and more than half $(53.4 \%, n=30)$ reported observing behaviours that were omitted from the current definitions of emergence.

Respondents were asked to provide free text answers describing what their teams do when they believe a patient has emerged, but they are unable to demonstrate via the recommended criteria. Forty-six respondents provided information from which four key themes were extracted; 1 ) continue assessment; 2 ) address additional problems; 3 ) negotiate a team decision; 4) the impact of uncertainty (Figure 2 ).

Figure 2: Themes arising from reports of what teams do if they believe a patient has emerged but are unable to demonstrate it (frequency of response in brackets). 


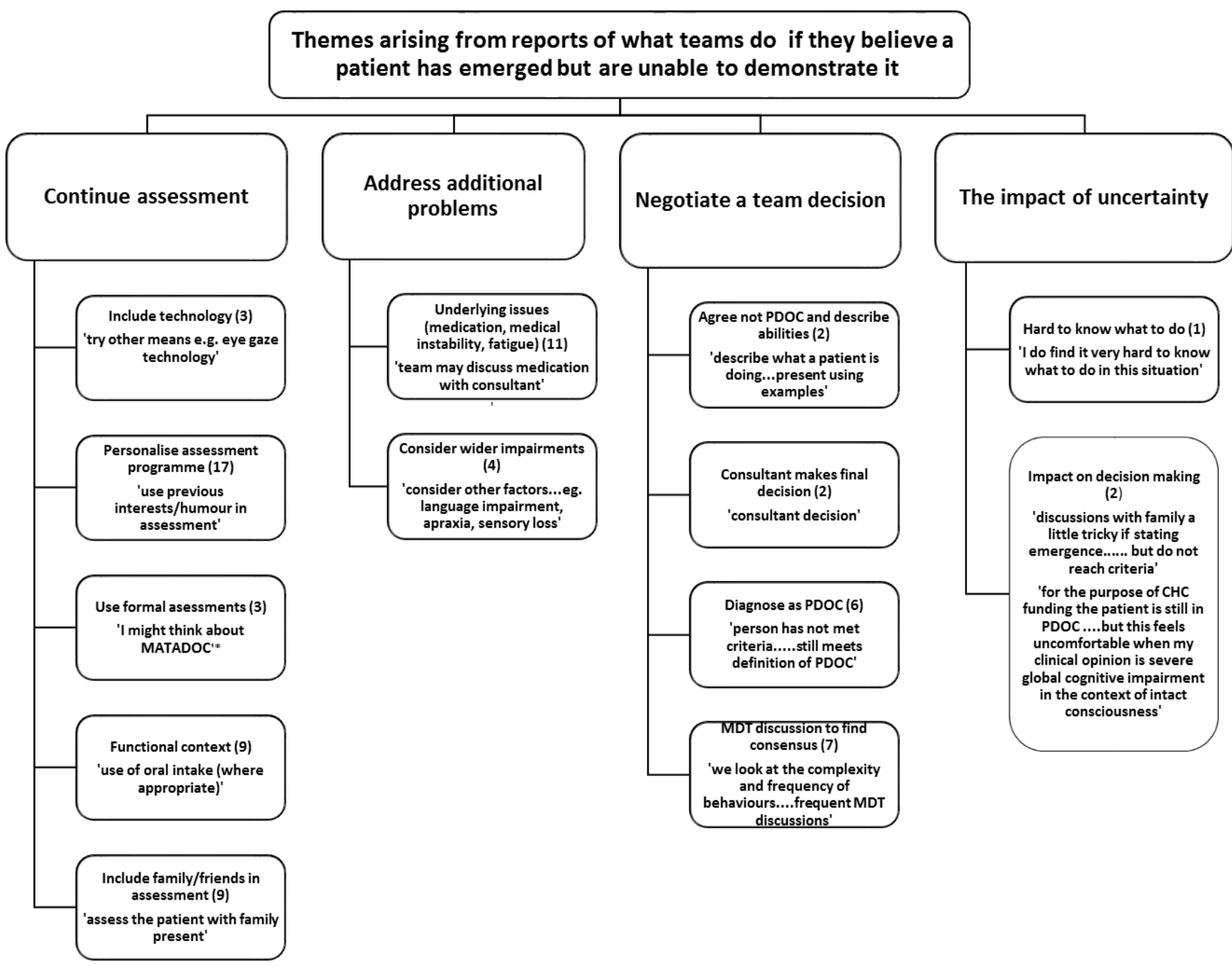

*MATADOC $=$ Music Therapy Assessment Tool for Awareness in Disorders of Consciousness (MATADOC) (Magee et al., 2014).

In response to an open question (Appendix I, Question 10), 29 respondents gave examples of behaviours that they observe which are incompatible with PDOC, but are not included in the current definitions of emergence. These were assigned to six key themes: 1) wider communication abilities; 2) appropriate emotional responses; 3) appropriate responses in functional tasks; 4) accepting responses at less than 100\% accuracy; 5) differentiating people in the environment; 6) lower level responses usually associated with VS or MCS (Figure 3).

Figure 3: Behaviours that respondents observe and believe to be incompatible with PDOC but not included in current definitions of emergence (frequency of response in brackets). 


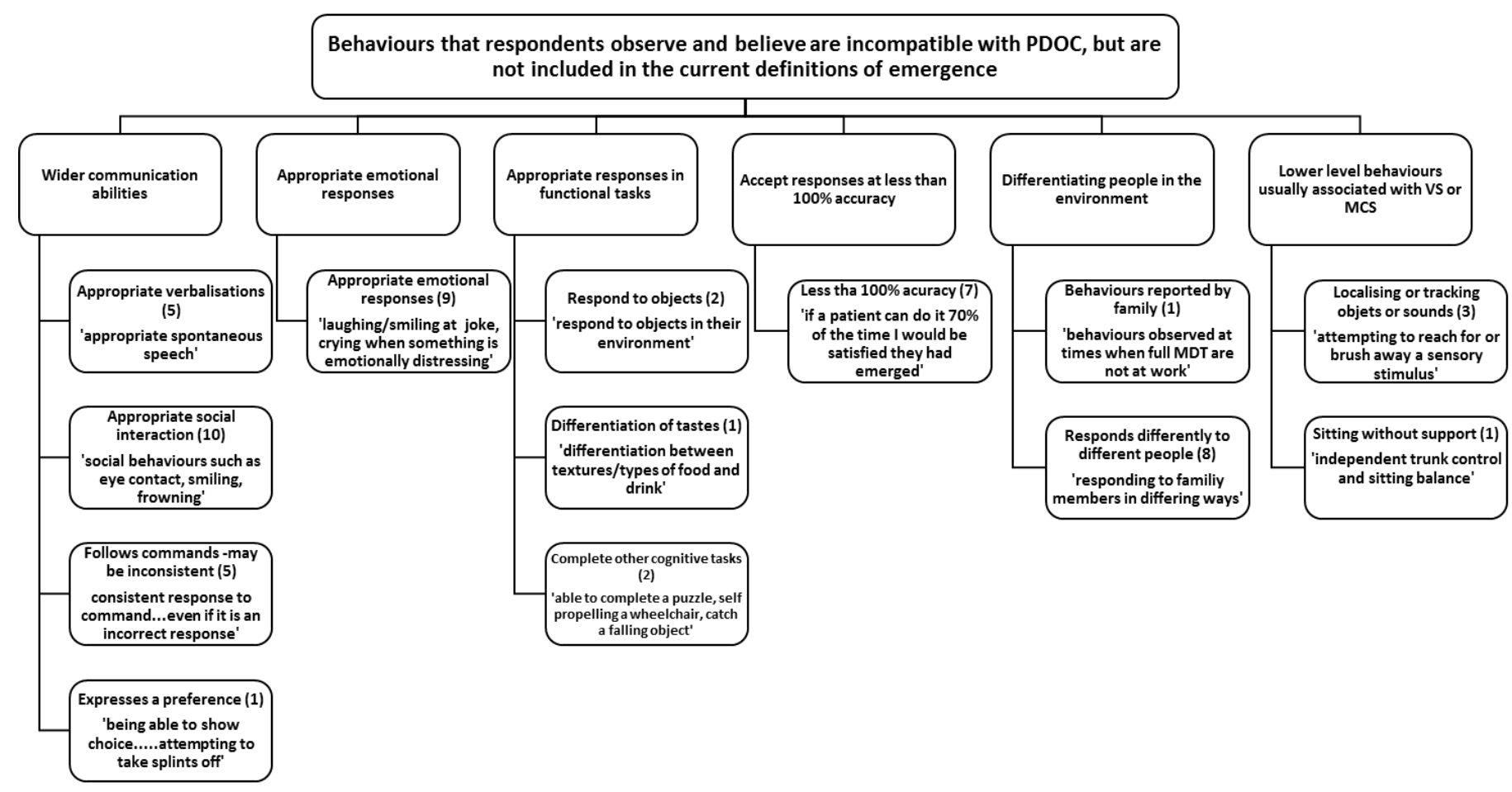

Participants were asked what they would do if a patient demonstrates some success on a range of tasks but does not achieve the required $100 \%$ accuracy on a single communication task. They were provided with four options: describe as MCS (23.2\% $n=13)$; describe as emerged $(8.9 \% \mathrm{n}=5)$; describe the patient's abilities without using a diagnostic label $(39.3 \%$, $\mathrm{n}=22$ ) and 'other' $(28.6 \%, \mathrm{n}=16)$. Respondents selecting 'other' were asked to indicate what they would do. Responses included: 'depends on context'; 'describe as emerging;' 'MCS plus a description of abilities'; 'would be dependent on whether a person had some success but did not hit the criteria (diagnose as MCS) or whether they hit the criteria but not consecutively (more likely to use emergence).'

\section{Assessment of functional communication}

Thirty-six participants answered questions on assessing functional communication to indicate emergence. Participants were asked if they regularly used the three recommended tasks. Yes/no questions were used by $100 \%(n=36)$ of participants, discrimination of two items by $88.9 \%(n=32)$ and matching pairs of items by $55.6 \%(n=20)$. Participants were also asked to indicate the type of stimuli they regularly use for each task (Figure 4).

Figure 4: Stimuli used for assessment of emergence 


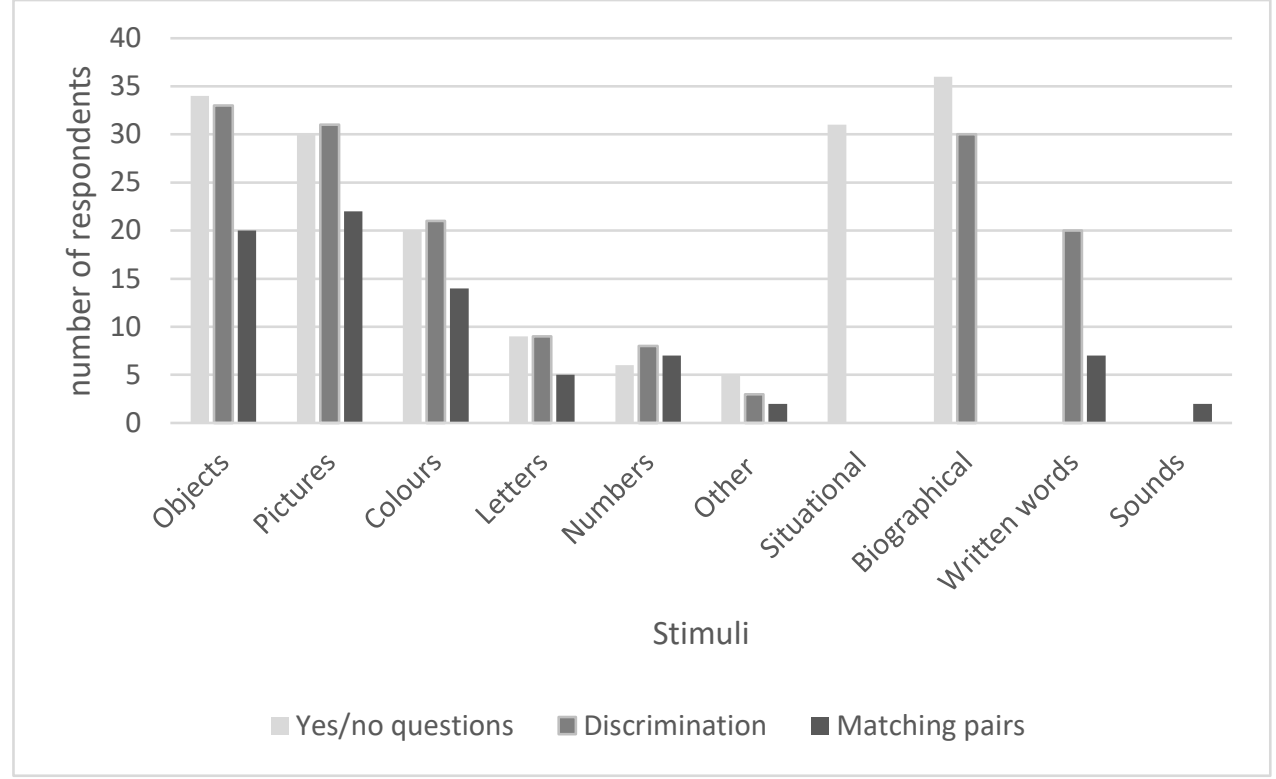

The most frequently-used stimuli for yes/no questions were: biographical information (e.g. is your name Joe?) used by $100 \%$ of respondents $(n=36)$, objects (e.g. Is this a cup?) (94.4\%, $\mathrm{n}=34$ ), situational (Am I pointing to the ceiling?) ( $86.1 \%, n=31$ ), and pictures (e.g. Is this a cat?) $(83.3 \%, n=30)$. Colours were also often used $(55.6 \%, n=20)$. Five respondents (13.9\%) employed other stimuli such as personal photos, items of personal interest, and the Putney Auditory Single Word Yes/No Assessment (PASWORD) (MacKenzie et al., 2006).

The most frequently used stimuli for discrimination of two items were objects $(91.7 \%, n=33)$, followed by pictures $(86.1 \%, n=31)$, biographical (e.g. family photographs) $(83.3 \%, n=30)$, and colours $(58.3 \%, \mathrm{n}=21)$. Three respondents $(8.3 \%)$ used other stimuli, including shapes or music.

The matching task was used least frequently, by only $55.6 \%(n=16)$ of respondents. The most frequently used stimuli for this task were pictures $(58.3 \%, n=22)$, objects $(52.8 \%, n=19)$, and colours $(38.9 \%, n=14)$. Matching sounds was used by two respondents $(5.6 \%)$.

All participants $(100 \%, n=36)$ were confident that they knew how to carry out a yes/no task correctly and the majority $(97.2 \%, n=35)$ were confident they could correctly carry out a discrimination task. However, there was less certainty about the matching task; $27.8 \%(n=10)$ disagreed that they were confident in its use. When asked if they were confident to use psycholinguistic principles to choose appropriate stimuli (e.g. ensuring items are phonologically, semantically and visually dissimilar), $69.4 \%(n=25)$ agreed they were confident to do so, but $30.6 \%(n=11)$ indicated a lack of confidence.

One third of respondents reported routinely using tasks other than the recommended ones to assess functional communication (33.3\%, $n=12)$. These tasks fell into four categories: 1 ) using high and/or low technology, such as eye gaze systems or switches; 2) assessing interaction with family present; 3 ) using personally relevant stimuli in a functional context 
(e.g. making choices in the kitchen); 4) considering 'lower level' behaviours, such as command-following and non-verbal communication (gesture, pointing and eye contact).

\section{Formal assessment}

Thirty-six respondents answered questions about the three formal assessments currently recommended by the RCP Guidelines (2013). The WHIM was used to determine emergence by $91.7 \%(n=33)$ of respondents, the CRS-R was used by $75.0 \%(n=27)$ and the SMART by $69.4 \%(n=25)$. Participants were given the option to indicate other formal assessments used in their setting. The Sensory Tool to Assess Responsiveness (STAR) (Stokes et al., 2018) and Music Therapy Assessment Tool for Awareness in Disorders of Consciousness (MATADOC) (Magee et al., 2014) were also used to determine emergence by $16.7 \%(n=6)$.

All 36 participants were asked to rate their confidence that each recommended assessment helps to determine emergence, whether they administered it themselves or not (Figure 5). Despite the WHIM being used by over $90 \%$ of respondents, only $19.4 \%(n=7)$ reported they were confident ('strongly agree/agree') that it helps determine emergence. Only $22.2 \%(n=8)$ expressed similar confidence in the CRS-R. The SMART was used less frequently but more respondents were confident that it helps to determine emergence $(41.7 \%, n=15)$. Thus frequency of use did not correspond with confidence that an assessment helps to determine emergence.

Figure 5: Respondents' confidence that a formal assessment helps to detect emergence from PDOC.

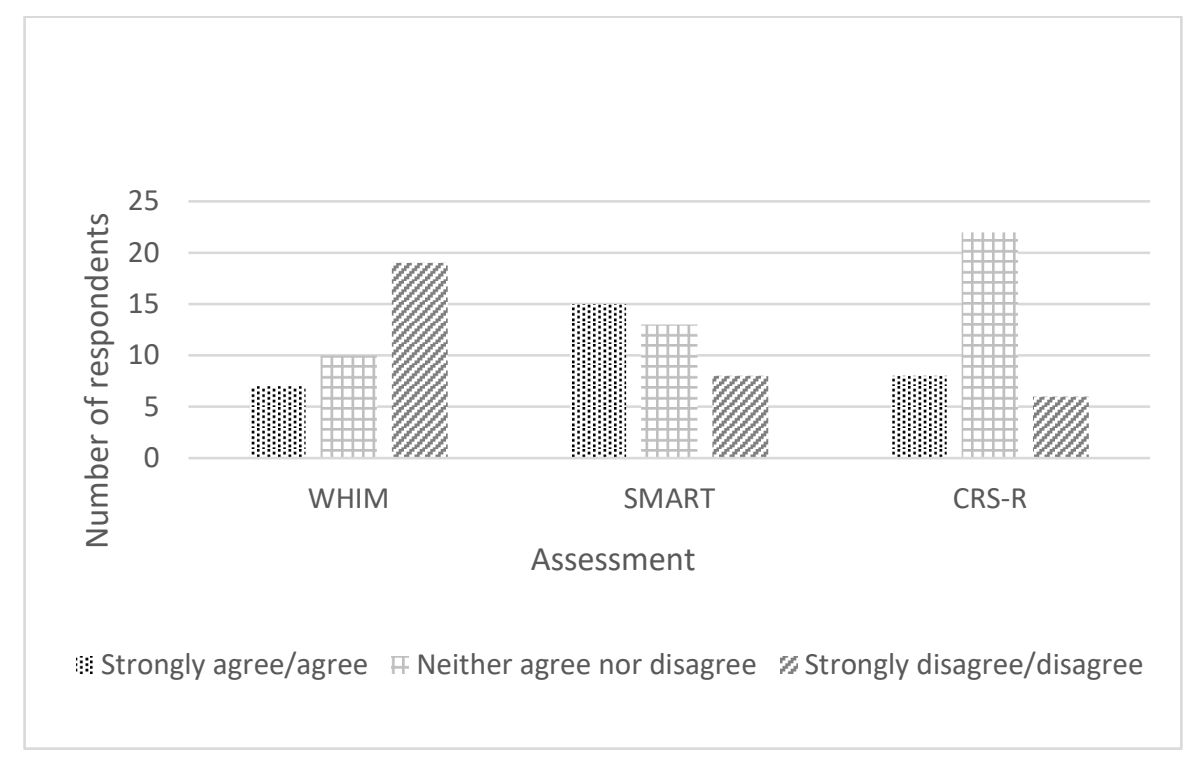

\section{Family involvement}

The majority of participants $(97.2 \%, n=35)$ reported involving families and friends in assessing functional communication in emerging patients. Respondents reported that families were involved in a variety of ways, including providing background information about the patient, 
and observing sessions and providing reassurance. Twenty eight respondents (80.0\%) involved family members in presenting specific tasks (Figure 6).

Figure 6: The involvement of families and friends in assessing functional communication to determine emergence

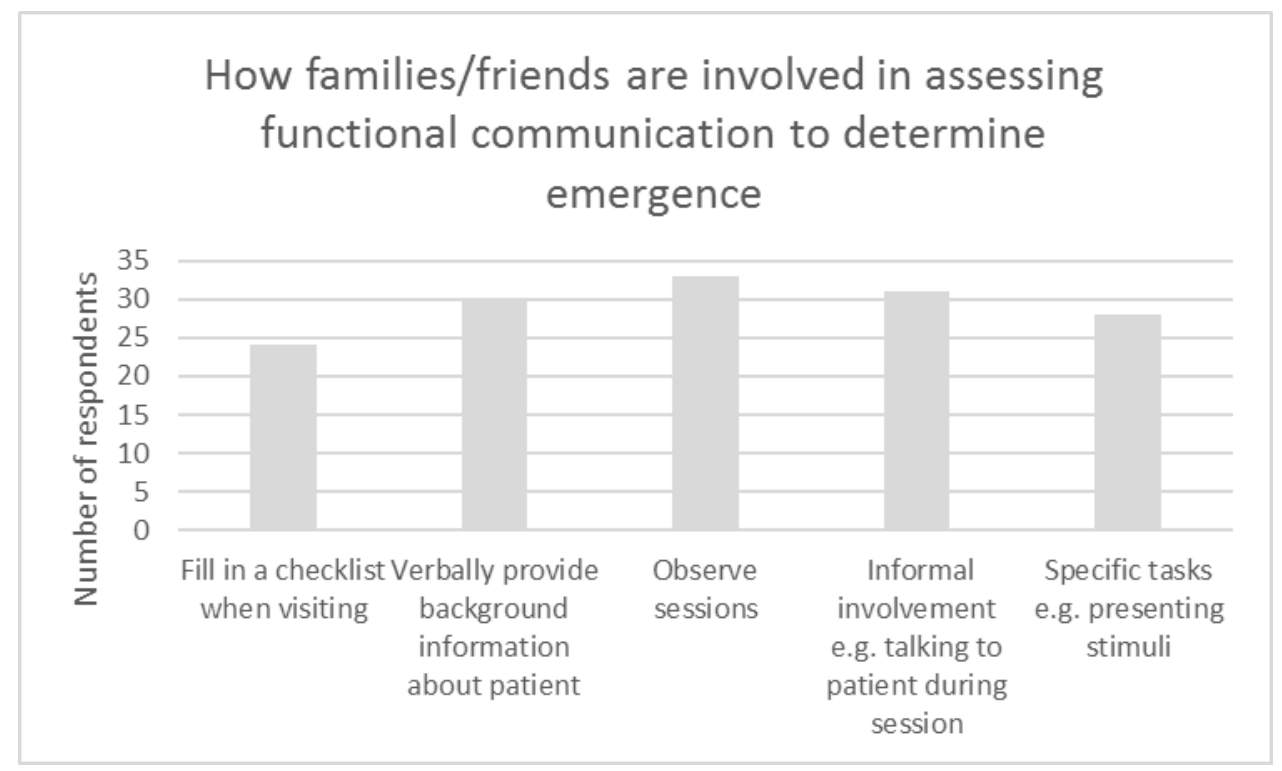

Respondents were asked open questions to explore what they considered the advantages and challenges of involving families and friends in the assessment of functional communication. Thirty six respondents provided information. Three key themes emerged around the advantages of family involvement: 1 ) it improves assessment; 2 ) it provides an opportunity for education; 3 ) it provides family with a valid role (Figure 7). Respondents felt that patients might be more likely to respond to a family member, and they also reported using information provided by family to choose more personally salient stimuli.

Figure 7: The advantages of involving families in assessing functional communication (frequency of response in brackets) 


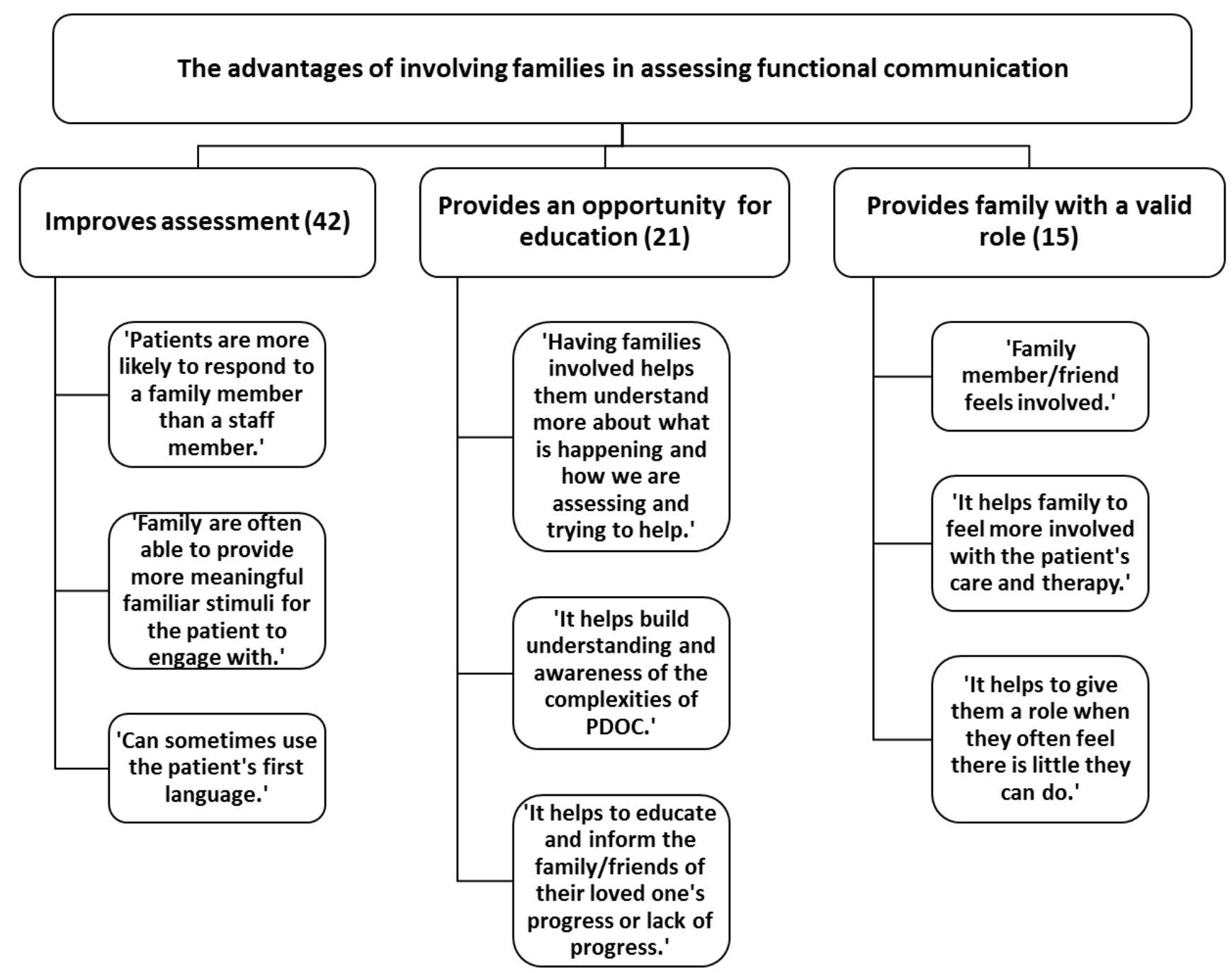

Questions regarding the challenges of including family and friends in the assessment of functional communication revealed five themes: 1 ) overestimation of patients' abilities; 2 ) prevention of assessment being carried out appropriately; 3 ) involvement can have a negative impact on the family; 4) additional time is required for education; 5) the impact on the relationship between the professional and the family (Figure 8). Respondents were concerned that relatives and friends had difficulty interpreting behaviours and overestimated a patient's abilities (for example, by interpreting a finger spasm as moving the finger purposefully), they could prevent accurate assessment by altering the assessment environment or being unable to administer tasks appropriately themselves, and they may find the emotional aspects of this involvement, and change of role, difficult. In addition, although respondents felt the opportunity to provide education was positive, it also presented a challenge due to the extra time taken.

Figure 8: The challenges of involving families in assessing functional communication (frequency of response in brackets). 


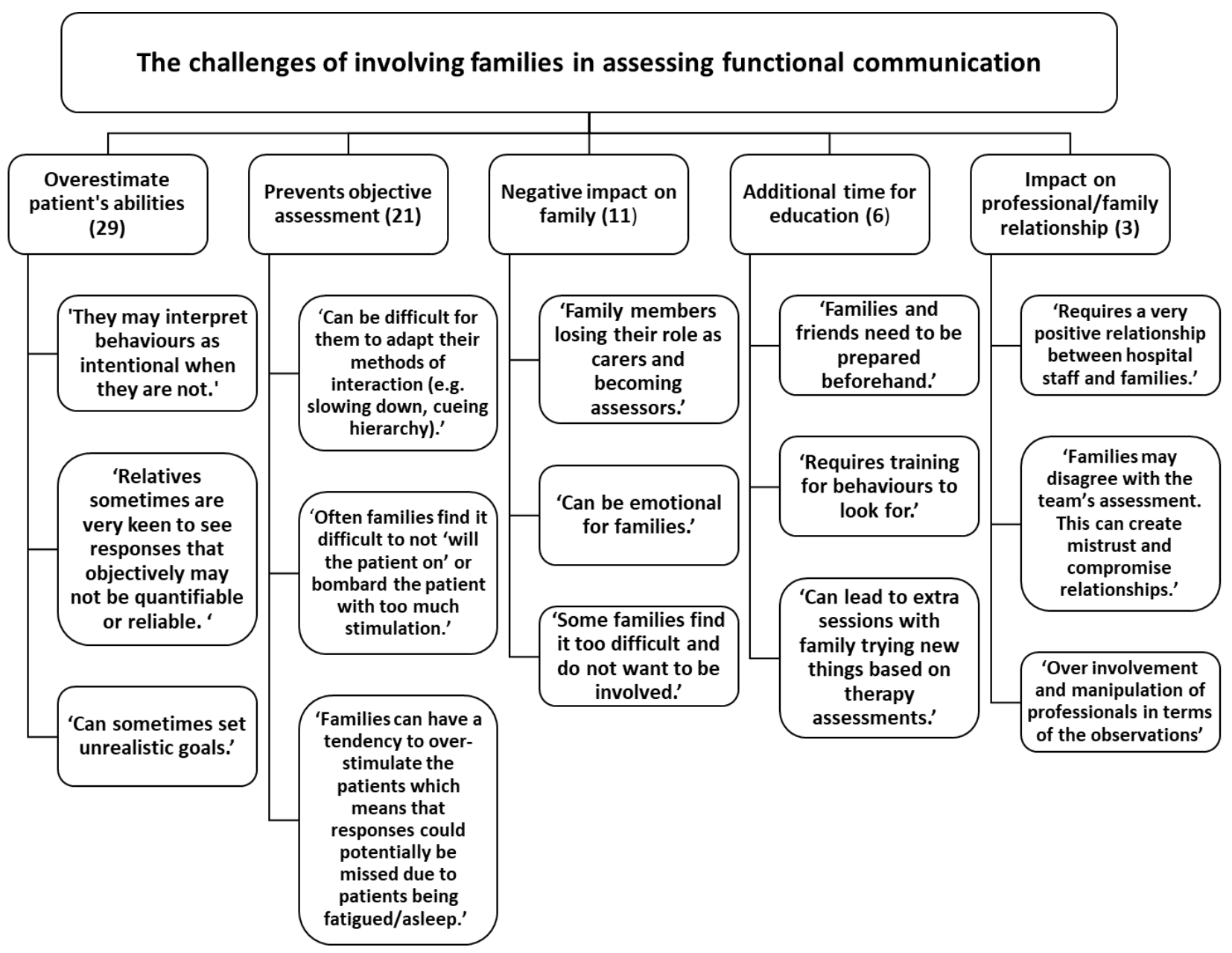

\section{Discussion}

This UK-wide survey was completed by a range of experienced professionals working in postacute assessment and rehabilitation settings with PDOC patients. The response rate was comparable to similar types of survey, and it is the only one to date exploring emergence from PDOC in the UK.

The findings highlight how current practice aligns with existing guidelines. A range of professionals were found to be involved in the assessment of emergence. Families were also involved and, for the majority of patients, diagnosis was established by the whole team. This reflects good practice in working with this complex population (RCP Guidelines, 2013). Clinicians reported using the recommended yes/no, discrimination and matching tasks, with varying amounts of confidence, to determine functional communication. Similarly, they used the recommended formal assessments to contribute to the assessment of emergence.

However, findings also indicate that a range of issues exist with assessing emergence from PDOC with the current recommended criteria and tasks (Giacino et al., 2002, RCP, 2013), and practice sometimes diverges from current recommendations. While many respondents used the recommended tasks, just under a third used additional tasks, such as observing interaction with familiar people. In addition, some respondents considered a range of 
observable behaviours, such as appropriate emotional responses, and accepted responses at less than the recommended $100 \%$ accuracy in judging that an individual had emerged from PDOC. Given that patients in PDOC have suffered a severe brain injury and are likely to have a range of cognitive and language impairments, or complications such as fatigue, arguably a 100\% accuracy criterion will be difficult to achieve (Dymoski et al., 2015, McDonald et al., 2014, Nakase-Richardson et al., 2008, Nakase-Richardson et al., 2009).

Respondents differed in their confidence levels concerning the use of recommended tasks to determine emergence, with almost half not confident that these could specifically detect the return of functional communication. Yes/no questions were used by all respondents, and discrimination of two items by the majority, to identify functional communication, with a reported high level of confidence in use of the tasks. In contrast, the matching task was only used by about half of respondents and over a quarter reported they would not be confident in carrying out this task. Guidance on how to set up this task was not provided in the 2013 RCP Guidelines.

The finding that approximately a third of respondents lacked confidence in choosing appropriate stimuli suggests clearer guidance is required. There are recommendations about possible stimuli (Giacino et al., 2002, RCP 2013), but respondents reported they use a wider range of items for yes/no, discrimination and matching tasks. The most popular were biographical information, objects, pictures and colours. Clinicians also made attempts to ensure stimuli were relevant to the individual e.g. using personal photographs for yes/no questions. The use of colours across all tasks could facilitate a response in patients with visual impairments affecting object and/or picture discrimination. Common objects are usually recognised by their shape, but impairments of visual fields, visual neglect and difficulties with eye movement are common after acquired brain injury (Zoltan, 2007), which may make blocks of colour easier to distinguish.

Clinicians reported using additional methods to detect the return of functional communication, including technology (such as low-tech switches or high-tech eye gaze systems), using personalized stimuli, assessing in a functional context, considering low level behaviours and assessing with family present. This practice is supported by evidence that patients in PDOC are more likely to respond to familiar and emotionally salient stimuli (Abbate et al., 2014; Luauté \& Perrin, 2015; Sun et al., 2018) and an enriched context (Di Stefano et al., 2012).

Respondents also carried out observation of behaviours that were considered to indicate emergence. These included verbal and non-verbal communication, such as inconsistent spontaneous speech in context, command following, gesture, appropriate eye contact, or smiling. They also noted differing responses to a range of people in the environment. These behaviours are currently considered to be consistent with MCS rather than emergence (Giacino et al. 2002). Command-following was identified by respondents as a potential indicator of emergence. Schnakers, et al. (2009) argued that this should be considered as a sign of emergence rather than MCS. However, Osborne et al. (2015) established a dissociation between command-following and communication, by demonstrating that patients who follow commands may be unable to use the movements functionally. For example, they can 
give a thumbs up to request, but cannot use the same movement for functional communication to indicate 'yes' or 'ok' in conversation. Pundole and Crawford (2017) suggested that if no single communication behaviour was $100 \%$ reliable, but communicative intent was observed each time other people are present, this could indicate consistent interaction and have implications for the diagnosis of emergence.

All three recommended formal assessments were used by survey respondents to help determine emergence, but confidence in their usefulness was low. The WHIM was used most frequently, followed by the CRS-R and then the SMART. Respondents indicated most confidence in the SMART, which could be as it is the only assessment to require specific training, but this was still fewer than half. The WHIM was not specifically designed for PDOC patients and allows for assessment of behaviour on a range of different tasks, which could explain its more widespread use. The CRS-R incorporates the specific criteria for emergence of functional object use and 6/6 situational yes/no questions, but a reported lack of confidence could originate from a reduced flexibility over choice of stimuli. In addition, despite the CRS-R being considered the 'gold standard' for PDOC assessment (Seel et al., 2010) there is evidence that results may be interpreted inconsistently (Chatelle et al., 2016). The SMART assesses yes/no, but also other cognitive tasks such as cause and effect using an auditory feedback switch, allowing some flexibility in assessment. This could account for why respondents were more confident that it could contribute to the assessment of emergence.

The majority of respondents reported that they worked with patients who they thought had emerged but were not able to meet the current criteria. Determining emergence is complex, and as consciousness is best considered as a continuum, it is likely to occur as a process rather than at a single point in time. Negotiating a team decision when diagnosis was unclear was not straightforward and these clinicians indicated that patients might be diagnosed as remaining in PDOC, as emerged, or by description of rather than a diagnostic label. There was no single approach, but some respondents indicated that decision making may be influenced by teams attempting to secure NHS continuing healthcare funding, which RCP guidelines (2013) state patients in PDOC should receive as a priority.

Benefits and challenges of involving family in the assessment of functional communication to indicate emergence were reported. The majority of respondents involved family in assessing functional communication, but this was usually in a support role, such as observing or providing background information, with only a fifth of respondents actively involving families in administering tasks. There is some evidence that asking family members to carry out formal assessments of patients in MCS may improve responses and change diagnosis (Sattin et al., 2014). However, some respondents felt families lacked objectivity and would misinterpret behaviours, provide extra cues or prevent assessment being carried out appropriately. Similar findings have been highlighted when comparing the assessment of communication in patients with severe aphasia by spouses and SLTs. According to Helmick et al. (1976) and Zraick \& Boone (1991) spouses considerably underestimated the severity of aphasia, resulting in unrealistic expectations. Such challenges have the potential to place a strain on the relationship between family members and clinicians, and need careful management when assessing emergence from PDOC. 
Respondents felt involving the family, although time consuming, could offer a valuable opportunity for education, and provide family members with a tangible role. Spouses of patients in PDOC can identify strongly with the role of caregiver, as previous roles change (Giovannetti et al., 2015), which might be useful during a time when they are experiencing significant loss and grief and are unsure how best to help (Soeterik et al., 2017). However, some respondents thought that blurring the role of family members from carer to assessor altered how they spent time with their loved one in a way that may be detrimental. This has also been found in partners of patients with severe aphasia who may take on the 'constraining and persistent role of caregiver' (Michallet, Le Dorze, \& Tétreault, 2001 p732). The views of family members on their involvement in the specific assessment of emergence are currently unknown.

\section{Limitations}

There are some limitations to the study, for example findings represent the views of clinicians in a post-acute setting so cannot be generalised to an acute or long term care environment. In addition, the questionnaire used was developed by the authors which could be considered to bias the questions asked. However care was taken to base questions on both a review of the literature and clinical expertise.

\section{Implications for further research and practice}

These findings suggest that the current emergence criteria and suggested tasks for determining the return of functional communication would benefit from review, as even experienced specialist teams, represented by these respondents, can find them hard to apply. There is currently some uncertainty about how to set up a matching task and how to choose appropriate stimuli. A recent update of the RCP guidelines (for publication 2020) has begun to address some of these issues. Respondents report using a wide range of stimuli and there is a clear need for further research to establish how tasks and stimuli impact on response accuracy in patients emerging from PDOC. The ways in which family and friends can optimally be involved in assessment also warrants future investigation. It is also necessary to determine whether expecting $100 \%$ accuracy is realistic in a severely brain injured population. Finally, there is a need for further guidance for clinical teams in how to deal with an uncertain diagnosis, as different approaches are currently applied.

Acknowledgements: This work was supported by The Royal Hospital for Neurodisability, London. Thank you to Dr. Sarah Crawford for comments on an earlier draft of this paper.

Declaration of Interests: The authors report no conflicts of interest.

\section{Word Count: 7875 (including tables, figures and references)}

\section{References}

Abbate, C., Trimarchi, P. D., Basile, I., Mazzucchi, A., \& Devalle, G. (2014). Sensory stimulation for patients with disorders of consciousness: from stimulation to rehabilitation. Frontiers in Human Neuroscience, 8(August), 1-5. 
https://doi.org/10.3389/fnhum.2014.00616

Barreca, S., Velikonja, D., Brown, L., Williams, L., Davis, L., \& Sigouin, C. S. (2003). Evaluation of the effectiveness of two clinical training procedures to elicit yes/no responses from patients with a severe acquired brain injury: A randomized single-subjects design. Brain Injury, 17(12), 1065-1075. https://doi.org/10.1080/0269905031000110535

Braun, Virginia; Clarke, V. (2013). Using thematic analysis in psychology. Journal of Chemical Information and Modeling, 53(9), 1689-1699.

https://doi.org/10.1017/СВ09781107415324.004

Chatelle, C., Bodien, Y. G., Carlowicz, C., Wannez, S., Charland-Verville, V., Gosseries, O., Laureys, S., Seel, R. T., \& Giacino, J. T. (2016). Detection and Interpretation of Impossible and Improbable Coma Recovery Scale-Revised Scores. Archives of Physical Medicine and Rehabilitation, 97(8), 1295-1300.e4.

https://doi.org/10.1016/j.apmr.2016.02.009

Di Stefano, C., Cortesi, A., Masotti, S., Simoncini, L., \& Piperno, R. (2012). Increased behavioural responsiveness with complex stimulation in VS and MCS: Preliminary results. Brain Injury, 26(10), 1250-1256.

https://doi.org/10.3109/02699052.2012.667588

Dymowski, A. R., Owens, J. A., Ponsford, J. L., \& Willmott, C. (2015). Speed of processing and strategic control of attention after traumatic brain injury. Journal of Clinical and Experimental Neuropsychology, 37(10), 1024-1035. https://doi.org/10.1080/13803395.2015.1074663

Eysenbach, G. (2004). Improving the quality of web surveys: The Checklist for Reporting Results of Internet E-Surveys (CHERRIES). Journal of Medical Internet Research, 6(3), 16. https://doi.org/10.2196/jmir.6.3.e34

Fins, J. J., Master, M. G., Gerber, L. M., \& Giacino, J. T. (2007). The Minimally Conscious State. Archives of Neurology, 64(10), 1400. https://doi.org/10.1001/archneur.64.10.1400

Giacino, J. T., Kalmar, K., \& Whyte, J. (2004). The JFK Coma Recovery Scale-Revised: Measurement characteristics and diagnostic utility. Archives of Physical Medicine and Rehabilitation, 85(12), 2020-2029. https://doi.org/10.1016/j.apmr.2004.02.033

Gill-Thwaites, H. (1997). The Sensory Modality Assessment Rehabilitation Technique - A tool for assessment and treatment of patients with severe brain injury in a vegetative state. Brain Injury, 11(10), 723-734. https://doi.org/10.1080/026990597123098

Giovannetti, A. M., Černiauskaite, M., Leonardi, M., Sattin, D., \& Covelli, V. (2015). Informal caregivers of patients with disorders of consciousness: Experience of ambiguous loss. Brain Injury, 29(4), 473-480. https://doi.org/10.3109/02699052.2014.990514

Kohl, A. D., Wylie, G. R., Genova, H. M., Hillary, F. G., \& DeLuca, J. (2009). The neural correlates of cognitive fatigue in traumatic brain injury using functional MRI. Brain Injury, 23(5), 420-432. https://doi.org/10.1080/02699050902788519

LeBlanc, J., de Guise, E., Feyz, M., \& Lamoureux, J. (2006). Early prediction of language impairment following traumatic brain injury. Brain Injury, 20(13-14), 1391-1401. 
https://doi.org/10.1080/02699050601081927

Luauté, J., \& Perrin, F. (2015). Can preferred music boost cognition in patients with disorders of consciousness? Annals of Physical and Rehabilitation Medicine, 58, e70. https://doi.org/10.1016/j.rehab.2015.07.171

MacKenzie, S., Gale, E., \& Munday, R. (2006). Putney Auditory Single Word Yes/No Assessment (PASWORD). Development of a reliable test of yes/no at a single word level in patients unable to participate in assessments requiring a specific motor response: An exploratory study. International Journal of Language and Communication Disorders, 41(2), 225-234. https://doi.org/10.1080/13682820500221436

Magee, W. L., Siegert, R. J., Daveson, B. A., Lenton-Smith, G., \& Taylor, S. M. (2014). Music Therapy Assessment Tool for Awareness in Disorders of Consciousness (MATADOC): Standardisation of the principal subscale to assess awareness in patients with disorders of consciousness. Neuropsychological Rehabilitation, 24(1), 101-124. https://doi.org/10.1080/09602011.2013.844174

McDonald, S., Gowland, A., Randall, R., Fisher, A., Osborne-Crowley, K., \& Honan, C. (2014). Cognitive factors underpinning poor expressive communication skills after traumatic brain injury: Theory of mind or executive function? Neuropsychology, 28(5), 801-811. https://doi.org/10.1037/neu0000089

Medicine, P., Society, B., Medicine, R., Society, C., \& Therapists, O. (2013). Prolonged disorders of consciousness National clinical guidelines. Royal College of Physicians.

Michallet, B., Le Dorze, G., \& Tétreault, S. (2001). The needs of spouses caring for severely aphasic persons. Aphasiology, 15(8), 731-747. https://doi.org/10.1080/02687040143000087

Nakase-Richardson, R., Yablon, S. A., Sherer, M., Nick, T. G. \& Evans, C. C., (2009). Emergence from minimally conscious state: Insights from evaluation of posttraumatic confusion. Neurology, 73(14), 1120-1126.

http://ovidsp.ovid.com/ovidweb.cgi?T=JS\&PAGE=reference\&D=emed9\&NEWS=N\&AN= 2009529483

Nakase-Richardson, R., Yablon, S. A., Sherer, M., Evans, C. C., \& Nick, T. G. (2008). Serial yes/no reliability after traumatic brain injury: Implications regarding the operational criteria for emergence from the minimally conscious state. Journal of Neurology, Neurosurgery and Psychiatry, 79(2), 216-218.

https://doi.org/10.1136/jnnp.2007.127795

Osborne, N. R., Owen, A. M., \& Fernández-Espejo, D. (2015). The dissociation between command following and communication in disorders of consciousness: an fMRI study in healthy subjects. Frontiers in Human Neuroscience, 9(September), 1-9. https://doi.org/10.3389/fnhum.2015.00493

Pundole, A., \& Crawford, S. (2017). The assessment of language and the emergence from disorders of consciousness. Neuropsychological Rehabilitation, 2011, 1-10.

https://doi.org/10.1080/09602011.2017.1307766 
Russell, K. C., Arenth, P. M., Scanlon, J. M., Kessler, L., \& Ricker, J. H. (2012). Hemispheric and executive influences on low-level language processing after traumatic brain injury. Brain Injury, 26(7-8), 984-995. https://doi.org/10.3109/02699052.2012.660513

Sattin, D., Giovannetti, A. M., Ciaraffa, F., Covelli, V., Bersano, A., Nigri, A., Ferraro, S., Minati, L., Rossi, D., Duran, D., Parati, E., \& Leonardi, M. (2014). Assessment of patients with disorder of consciousness: do different Coma Recovery Scale scoring correlate with different settings? Journal of Neurology, 261(12), 2378-2386. https://doi.org/10.1007/s00415-014-7478-5

Schaeffer, N. C., \& Dykema, J. (2011). Questions for surveys: Current trends and future directions. Public Opinion Quarterly, 75(5 SPEC. ISSUE), 909-961. https://doi.org/10.1093/poq/nfr048

Schnakers, C., Bessou, H., Rubi-Fessen, I., Hartmann, A., Fink, G. R., Meister, I., Giacino, J. T., Laureys, S., \& Majerus, S. (2015). Impact of aphasia on consciousness assessment: A cross-sectional study. Neurorehabilitation and Neural Repair, 29(1). https://doi.org/10.1177/1545968314528067

Schnakers, C., Majerus, S., Giacino, J. T., \& Laureys, S. (2009). The problem of aphasia in the assessment of consciousness in brain-damaged patients \$. 177(1), 49-61. https://doi.org/10.1016/S0079-6123(09)17705-1

Seel, R. T., Sherer, M., Whyte, J., Katz, D. I., Giacino, J. T., Rosenbaum, A. M., Hammond, F. M., Kalmar, K., Pape, T. L. B., Zafonte, R., Biester, R. C., Kaelin, D., Kean, J., \& Zasler, N. (2010). Assessment scales for disorders of consciousness: Evidence-based recommendations for clinical practice and research. Archives of Physical Medicine and Rehabilitation, 91(12), 1795-1813. https://doi.org/10.1016/j.apmr.2010.07.218

Shiel, A., Horn, S. A., Wilson, B. A., Watson, M. J., Campbell, M. J., \& McLellan, D. L. (2000). The wessex head injury matrix (WHIM) main scale: A preliminary report on a scale to assess and monitor patient recovery after severe head injury. Clinical Rehabilitation, 14(4), 408-416. https://doi.org/10.1191/0269215500cr326oa

Slovarp, L., Azuma, T., \& Lapointe, L. (2012). The effect of traumatic brain injury on sustained attention and working memory. Brain Injury, 26(1), 48-57. https://doi.org/10.3109/02699052.2011.635355

Soeterik, S. M., Connolly, S., Playford, E. D., Duport, S., \& Riazi, A. (2017). The psychological impact of prolonged disorders of consciousness on caregivers: A systematic review of quantitative studies. Clinical Rehabilitation, 31(10), 1374-1385. https://doi.org/10.1177/0269215517695372

Stokes, V., Gunn, S., Schouwenaars, K., \& Badwan, D. (2018). Neurobehavioural assessment and diagnosis in disorders of consciousness: a preliminary study of the Sensory Tool to Assess Responsiveness (STAR). Neuropsychological Rehabilitation, 28(6), 966-983. https://doi.org/10.1080/09602011.2016.1214604

Sun, Y., Wang, J., Heine, L., Huang, W., Wang, J., Hu, N., Hu, X., Fang, X., Huang, S., Laureys, S., \& Di, H. (2018). Personalized objects can optimize the diagnosis of EMCS in the 
assessment of functional object use in the CRS-R: A double blind, randomized clinical trial. BMC Neurology, 18(1), 1-5. https://doi.org/10.1186/s12883-018-1040-5

Turk, T., Elhady, M. T., Rashed, S., Abdelkhalek, M., Nasef, S. A., Khallaf, A. M., Mohammed, A. T., Attia, A. W., Adhikari, P., Amin, M. A., Hirayama, K., \& Huy, N. T. (2018). Quality of reporting web-based and non-web-based survey studies: What authors, reviewers and consumers should consider. PLOS ONE, 13(6), 1-15.

https://doi.org/10.1371/journal.pone.0194239 